\title{
Adecuación de la disponibilidad farmacológica en un Servicio de Medicina Interna a la lista de medicamentos esenciales de la OMS
}

\author{
J. A. DÍAZ PEROMINGO, I. VILLAMIL CAJOTO, L. BUJÁN DE GONZALO' \\ Servicios de Medicina Interna $y^{\prime}$ Farmacia. Hospital da Barbanza. Riveira. A Coruña
}

\section{RESUMEN}

Introducción: Desde hace años la organización mundial de la salud (OMS) elabora una lista de medicamentos esenciales (LME), considerando como tales a los que cubren las necesidades de atención de salud prioritarias de la población. Son seleccionados según su importancia, eficacia, inocuidad y relación costo-eficacia. No existe información suficiente sobre la adecuación de esta lista al uso habitual en la práctica común en Medicina Interna.

Material y métodos: Se comparó la LME de la OMS (13 a edición, revisada en abril de 2003) con los utilizados en el Servicio de Medicina Interna del Hospital da Barbanza, Riveira, A Coruña (España), Fundación Pública del Servizo Galego de Saúde en el período 2004-2005.

Resultados: De los 27 grupos de la lista de la OMS se excluyeron 2 porque su uso no es habitual en Medicina Interna. De los restantes 25 grupos, se encontraron diferencias en 24 grupos. En 1 grupo ambas listas eran idénticas. En los 24 grupos con diferencias se usaban menos fármacos de los recomendados por la OMS. Se analizaron 11 grupos; aquéllos en los que el número de diferencias era mayor que 5. Las causas resultaron ser: grupos 6,8 y 12 , fármacos para pacientes habitualmente remitidos a nuestro hospital de referencia; fármacos no utilizados habitualmente en Medicina Interna (grupos 1,4,13,14,18,19 y 21) y medicamentos que no cubre el Sistema Nacional de Salud (Grupo 27).

Conclusiones: La valoración de la adecuación de los fármacos utilizados en Medicina Interna en un Hospital Comarcal muestra un uso significativamente inferior a las recomendaciones estándar de la OMS. Las causas identificadas son terapias no cubiertas por un Centro como el nuestro y aquellos fármacos no utilizados habitualmente en Medicina Interna. La OMS estima que unos 2000 millones de personas no tienen acceso regular a medicamentos esenciales y vacunas de calidad en el mundo. La dimensión del problema trasciende el ámbito de la salud, suponiendo un problema social, económico y ético por lo que el buen uso de los fármacos esenciales en sistemas sanitarios desarrollados es un buen ejemplo de eficiencia.

PALABRAS CLAVE: Medicamentos esenciales. Salud pública. Políticas de salud. Economía de la Medicina.

\author{
ADEQUACY OF THE PHARMACOLOGICAL DISPONIBILITY IN A \\ DEPARTMENT OF INTERNAL MEDICINE TO THE WHO ESSENTIAL \\ DRUG LIST
}

\section{ABSTRACT}

Introduction: Since several years ago, the World Health Organization (WHO), publishes the Essential Drug List (EDL) including medicines that are used to cover the mayor health community problems. Drugs are selected in terms of importance, usefulness, safety and cost-effectiveness. There is no enough data about adequacy between this list and the current medical practise in Internal Medicine.

Material and methods: Both EDL (13 edition, reviewed on April 2003) and the list of medicines used in the Internal Medicine Department from the Hospital da Barbanza, Riveira, A Coruña (Spain), Fundación Pública del Servizo Galego de Saúde in 2004-2005 were compared.

Results: From the 27 groups included in the EDL, 2 groups were excluded because no regular use in Internal Medicine. Among the other 25 groups there were significant differences in 24 groups. Just in one group both lists were identical. In all the 24 groups with differences, we used an smaller amount of drugs that recommended in the EDL. We analysed 15 groups (the groups with at least 5 differences between both lists). Causes identified were as follows: drugs used in diseases that we used to send to another hospital (Reference Hospital) in groups 6,8 and 12; drugs not commonly prescribed in Internal Medicine in groups $1,4,13,14,18,19$ and 21 , and finally drugs not currently financed by the National Health System (group 27).

Conclusions: After examining the adequacy of the list of drugs prescribed in Internal Medicine in a Community Hospital we conclude that its use is significantly lower than the standard recommendations derived from de WHO. Identified causes are treatments not covered in a Community Hospital needing to transfer patients to another hospital and the inclusion of drugs not commonly used in Internal Medicine. The WHO estimates that over 2000 million people can not currently access to essential drugs nor vaccines all over the world. The problem is not just a health problem but also a social, economic and ethic one. So, an accurate use of such essential drugs in developed Health Systems could be a good example of efficiency.

KEY WORDS: Essential drugs. Public health. Health policy. Economics of medicine.

Díaz Peromingo JA, Villamil Cajoto I, Buján de Gonzalo L. Adecuación de la disponibilidad farmacológica en un Servicio de Medicina Interna a la lista de medicamentos esenciales de la OMS. An Med Interna (Madrid) 2007; 24: 173-176.

\section{INTRODUCCIÓN}

La Organización Mundial de la Salud (OMS) y la comunidad internacional han estado de acuerdo a lo largo de las últi- mas décadas en numerosas resoluciones y declaraciones que afectaban a las políticas farmacéuticas, políticas de salud y estrategias de salud. En el ánimo de estas actuaciones están conceptos tan amplios y aceptados como el acceso equitativo

Trabajo aceptado: 18 de diciembre de 2006 
a la asistencia sanitaria, la calidad de los servicios y el control de la escalada de costes ante la limitación de los recursos. La aplicación de estos conceptos se debe realizar a varios niveles, entre ellos el hospitalario. En los últimos años, tanto la declaración de Alma-Ata de 1978 sobre "Salud para todos para el año 2000" (Health for all by the year 2000) (1) como las "Metas para la cumbre del milenio (Millenium Summit Goals), celebrada en la Organización de las Naciones Unidas (ONU) en septiembre del año 2000, redundan en la necesidad de cooperación por parte de los sistemas de salud y de las compañías farmacéuticas para proveer un acceso sostenible a los medicamentos esenciales en los países en vías de desarrollo.

Debido al impacto que tiene sobre la calidad asistencial y sus costes la selección de los medicamentos considerados básicos para la asistencia sanitaria, la OMS ha publicado una Lista de Medicamentos Esenciales (LME); dicha lista se revisa periódicamente y constituye una herramienta muy importante en el intento de asegurar la disponibilidad general de este tipo de fármacos. El acceso a los medicamentos esenciales debe además cumplir con los requerimientos de calidad, seguridad y coste-efectividad, para alcanzar el objetivo del uso racional de los mismos. Idealmente esta elección debería basarse en la evidencia científica y estar libre de presiones comerciales. En este sentido, la actual LME de la OMS busca precisamente esto al combinar guías terapéuticas y revisiones sistemáticas de la evidencia clínica que existe para cada tratamiento. El concepto y los principios que guían a esta LME de la OMS no sólo son aplicables a los países en vías de desarrollo sino también a los industrializados, afectando así a la selección básica de los medicamentos que se usan tanto en el ámbito hospitalario como en atención primaria.

En el presente estudio se compara la LME de la OMS con los medicamentos que constituyen el arsenal terapéutico del Servicio de Medicina Interna en un Hospital Comarcal, para evaluar las posibles discrepancias y estudiar sus causas.

\section{MATERIAL Y MÉTODOS}

Se realizó una comparación entre la LME de la OMS, según la última versión revisada del año 2003 que define 27 grupos de medicamentos considerados esenciales (Tabla I), y una relación de los medicamentos prescritos a los pacientes ingresados en el Servicio de Medicina Interna de un Hospital Comarcal durante el período 2004-2005. Se analizaron las diferencias encontradas entre ambos listados y se investigaron sus posibles causas. Se analizaron pormenorizadamente aquéllos grupos en los que el número de diferencias era igual o mayor a cinco.

\section{RESULTADOS}

De los 27 grupos que están definidos en la lista de la OMS (Tabla I), se excluyeron el 22 (Oxitócicos y Antioxitócicos) y el 23 (Solución de Diálisis Peritoneal), por incluir medicamentos que no son de uso habitual en la práctica clínica de la especialidad de Medicina Interna.

De los 25 grupos restantes, sólo en uno -el 9- correspondiente a los Antiparkinsonianos, existía total coincidencia entre ambas listas; mientras que en los 24 grupos que presentaban discrepancias, el listado de medicamentos que usaba el Servicio de Medicina Interna contenía menos fármacos que el listado de la OMS.
TABLA I

GRUPOS FARMACOLÓGICOS INTEGRANTES DEL LISTADO DE MEDICAMENTOS ESENCIALES DE LA OMS

1. Anestésicos

2. Analgésicos, antipiréticos, antiinflamatorios no esteroideos (AINE), antigotosos y antirreumáticos modificadores de la enfermedad (ARME)

3. Antialérgicos y medicamentos usados en la anafilaxia.

4. Antídotos y otras sustancias usadas en las intoxicaciones

5. Anticonvulsivantes/antiepilépticos

6. Antiinfecciosos

7. Antimigrañosos

8. Antineoplásicos, inmunosupresores y medicamentos usados en los cuidados paliativos

9. Antiparkinsonianos

10. Medicamentos que afectan a la sangre

11. Productos sanguíneos y sucedáneos del plasma

12. Medicamentos cardiovasculares

13. Medicamentos dermatológicos (tópicos)

14. Agentes de diagnóstico

15. Desinfectantes y antisépticos

16. Diuréticos

17. Medicamentos gastrointestinales

18. Hormonas, otros medicamentos endocrinos y anticonceptivos

19. Productos inmunológicos

20. Miorrelajantes (de acción periférica) e inhibidores de la colinesterasa

21. Preparaciones oftalmológicas

22. Oxitócicos y antioxitócicos

23. Solución de diálisis peritoneal

24. Medicamentos psicoterapéuticos

25. Medicamentos que actúan en las vías respiratorias.

26. Soluciones correctoras de los trastornos hidroelectrolíticos y del equilibrio acidobásico

27. Vitaminas y minerales

\section{TABLA II}

GRUPOS ANALIZADOS POR PRESENTAR UN NÚMERO DE DIFERENCIAS MAYOR O IGUAL A 5 ENTRE AMBOS LISTADOS

1. Anestésicos

4. Antídotos y otras sustancias usadas en las intoxicaciones

6. Antiinfecciosos

8. Antineoplásicos, inmunosupresores y medicamentos usados en los cuidados paliativos

12. Medicamentos cardiovasculares

13. Medicamentos dermatológicos (tópicos)

14. Agentes de diagnóstico

18. Hormonas, otros medicamentos endocrinos y anticonceptivos

19. Productos inmunológicos

21. Preparaciones oftalmológicas

27. Vitaminas y minerales 
Se identificaron 11 grupos con al menos 5 diferencias entre ambas listas (Tabla II). Para los grupos 6, 8 y 12, la causa que las explica fue que estos grupos contienen fármacos que se usan fundamentalmente para patologías que no se tratan en nuestro centro y son trasladadas a un hospital de referencia. En los grupos 1, 4, 13, 14, 18, 19 y 21 se identificó como probable origen de disparidad el hecho de que contienen medicamentos usados con poca frecuencia, en general, en un Servicio de Medicina Interna. En el caso del grupo 27, la razón de las diferencias sería el hecho de que incluye medicamentos que en la actualidad no se encuentran financiados por el Sistema Nacional de Salud. El resumen de estas discrepancias se expone en la tabla III.

\section{DISCUSIÓN}

El concepto de medicamentos esenciales fue introducido por primera vez en la World Health Assembly en 1975, publicándose en 1977 la primera Lista de Medicamentos Esenciales que debe ser y es adaptada de forma continua a un mundo cambiante con nuevos fármacos, nuevas indicaciones y nuevas enfermedades (2). Las recomendaciones del Comité de Expertos responsable de la actualización de la LME de la OMS incluyen las consideraciones y justificaciones de dicho Comité para añadir o quitar medicamentos de la lista y anexos como la última versión revisada del modelo de LME de la OMS $\left(13^{a}\right.$ edición) $(3,4)$. Se ha intentado también clasificar los medicamentos de administración oral de la Lista de Medicamentos Esenciales de acuerdo con el sistema de clasificación biofarmacéutica que se implantó por primera vez en 1995 sin evidenciarse una total coincidencia entre ambos sistemas (5).

A nivel hospitalario, una de las comisiones clínicas de obligada existencia es la de Farmacia y Terapéutica (CFT) que se ocupa de la selección consensuada de un conjunto de medicamentos que, de acuerdo con los conocimientos del momento, satisfaga la mayor parte de las necesidades clínicas del centro. Dicha selección debe quedar reflejada en la elaboración de una Guía Farmacoterapéutica. La CFT está constituida por un equipo multidisciplinar de profesionales que debe valorar diversos aspectos a la hora de incluir o excluir un medicamento de la guía como son: aportación del fármaco al arsenal terapéutico (evitando duplicidades), eficacia, perfil toxicológico, costes (de adquisición, de preparación...), comodidad de administración, pre- sentaciones comerciales (envase clínico, acondicionamiento en dosis unitarias), requerimientos de espacio en el almacenamiento, laboratorio investigador, repercusión en el área sanitaria, aspectos farmacocinéticos... Las prescripciones de los facultativos de los distintos Servicios Clínicos deben adaptarse, en la medida de lo posible, a esta selección. La variedad de medicamentos que se usa de forma habitual en un Servicio de Medicina Interna de un Hospital Comarcal es significativamente inferior a las recomendaciones estándar de la LME de la OMS. Las principales causas, identificadas en nuestro estudio, que explican la discordancia entre ambos listados son el escaso uso en nuestro Servicio de ciertos fármacos empleados en el tratamiento de patologías concretas que se derivan a un centro de referencia, el tratarse de fármacos poco usados en un Servicio de Medicina Interna o el haber sido excluidos de financiación por parte del Sistema Nacional de Salud.

En algunos subgrupos existen otras causas de discrepancias como, por ejemplo, en el de los medicamentos cardiovasculares (grupo 12), en particular en el subgrupo de los antihipertensivos, que en la lista de la OMS consta de 6 medicamentos (atenolol, enalapril, hidralazina, hidroclorotiazida, metildopa y nifedipino) y en nuestro centro contamos con 10 (atenolol, captopril, enalapril, hidralazina, hidroclorotiazida, metildopa, nifedipino, amlodipino, losartán y valsartán). Creemos que esta discordancia puede deberse a la gran importancia que se presta a la hipertensión en los países industrializados y a la investigación y presión farmacéutica existente en este campo. En este grupo 12, de medicamentos cardiovasculares, existen fármacos de uso en Unidades Coronarias o de Cuidados Intensivos que nuestro Centro no posee y que justifica en gran parte estas diferencias.

El caso del grupo 6 (Antiinfecciosos) también requiere un análisis más detallado. Los antibacterianos están en una relación prácticamente de 2:1 (47 lista OMS versus 23 en nuestra lista). Los antihelmínticos y los repelentes de insectos no están representados en nuestro Centro. Los antifúngicos suponen 6 en la lista OMS y 1 en nuestro hospital. Los antivirales son 13 en la lista OMS versus 1 en nuestro centro, fundamentalmente por el hecho de que los pacientes con infección crónica por el VIH reciben su tratamiento ambulatorio en un hospital de referencia. Así pues, dentro de los antivirales, son los antiretrovirales los principales causantes de las discrepancias encontradas. Los antiprotozoarios son 28 en la lista OMS y 2

\section{TABLA III}

CAUSAS Y GUPOS DE DISCREPANCIA ENTRE AMBOS LISTADOS

\begin{tabular}{lccc}
\hline Causas & $\begin{array}{c}\text { Derivación a } \\
\text { otro centro }\end{array}$ & $\begin{array}{c}\text { Uso poco habitual en } \\
\text { Medicina Interna }\end{array}$ & $\begin{array}{c}\text { Exclusión del sistema } \\
\text { nacional de salud }\end{array}$ \\
\hline GRUPOS: & $6,8,12$ & $1,4,13,14,18,19,21$ & 27 \\
EJEMPLOS & Antihelmínticos & Anestésicos generales & Vitaminas y minerales \\
& Antivirales & Antídotos & Medicamentos que afectan \\
Antiprotozoarios & a la diferenciación y proliferación \\
& Citotóxicos & cutáneas \\
& Medios de contraste radiológico & Anticonceptivos \\
& Vacunas \\
\end{tabular}


en la nuestra probablemente por la baja prevalencia de estas infecciones en nuestra área sanitaria.

La estrategia actual de la OMS se basa en mejorar el acceso a los medicamentos esenciales, fortalecer las políticas farmacéuticas nacionales, la calidad y seguridad de los medicamentos y fomentar su uso racional. Todos los países se enfrentan en mayor o menor grado a los mismos problemas básicos y retos. Es necesario buscar el acuerdo en cuanto a las políticas farmacéuticas para cada país y, a inferior escala, para cada institución, y diseñar estrategias de actuación que impliquen la obtención de indicadores de eficacia que resulten fáciles de medir y sean comparables. Los países y los hospitales pueden diferir en parte pero la base debería ser común $(6,7)$. En algunos países como Brasil o Méjico se ha estudiado la disponibilidad de medicamentos esenciales dentro del sistema público de salud, observándose que ésta es baja y tiene una amplia variabilidad, y comprobándose que las personas que suelen necesitar esas medicinas son precisamente las que no tienen acceso a ellas, ya que las oficinas de Farmacia privadas suelen ser la principal fuente de medicamentos esenciales y el precio de los mismos es muy elevado $(8,9)$.

El interés por la disponibilidad de los medicamentos esenciales, si bien tuvo su punto álgido en los años 70 y 80 del siglo pasado, cuando la Sanidad Pública presionó a la Industria Farmacéutica para que aceptase este concepto, ha vuelto a resurgir y es un tema de nuevo candente habida cuenta de que la Lista de Medicamentos Esenciales de la OMS forma parte de la política farmacéutica nacional de 146 países. El debate en los aspectos relacionados con el comercio, los derechos de propiedad intelectual, las patentes y el acceso a los medicamentos causa preocupación en los sistemas públicos de salud (10).

Este primer listado ayudó a establecer el principio de que algunas medicinas eran más útiles que otras y a comprobar que las esenciales eran con frecuencia inaccesibles para muchas poblaciones (11). A la definición de medicamentos esenciales se fueron añadiendo criterios como su relevancia para la salud pública, la optimización del balance coste-efectividad y la minimización de los riesgos asociados a su uso.
Además la OMS modificó los criterios de inclusión en la LME por que hasta 2002 uno de los criterios era el precio de los medicamentos, lo que dejaba fuera de la lista medicamentos esenciales pero caros.

Algunos autores preconizan que se debe ir más allá que el enfoque tradicional de la eficacia y la seguridad avanzando hacia la evidencia de buena calidad. Así la inclusión de nuevos medicamentos en la Lista de Medicinas Esenciales debería cumplir una serie de criterios predeterminados y la retirada debería hacerse sólo si se dispone de alternativas superiores (12). Tanto la igualdad en el acceso como el uso racional de los medicamentos esenciales llevan a la OMS a levantar su voz en los acuerdos comerciales internacionales y a investigar en el desarrollo de estrategias que garanticen la disponibilidad de estos fármacos allí donde se necesitan (13). En países como Sudáfrica las prescripciones de los Médicos Generales diferían sustancialmente de la Lista de Medicamentos Esenciales "adaptada" por el Departamento de Salud de ese país a las necesidades existentes allí originando un debate en torno al porqué de estas diferencias tan importantes; de hecho, sólo el 22,4\% de las prescripciones, en todas las áreas estudiadas se ajustaban a dicha lista (14).

Algunos autores han planteado, a lo largo de las sucesivas revisiones que ha tenido esta lista, si los fármacos añadidos que siempre van superando a los retirados eran realmente medicamentos esenciales en cuanto a estar en relación con nuevas entidades clínicas o nuevas indicaciones o si muchas de las incorporaciones son debidas a cambios en la percepción sobre la utilidad de un determinado fármaco y no a un avance científico significativo (15).

Tanto los profesionales médicos como los farmacéuticos ocupan una posición única en la tarea de establecer las actuaciones prioritarias a llevar a cabo en cada centro hospitalario en el ámbito de la política farmacéutica. La colaboración multidisciplinar es fundamental para detectar, prevenir o resolver los problemas relacionados con los medicamentos y su uso prestando especial atención a las indicaciones, contraindicaciones, interacciones y efectos adversos.

\section{Bibliografía}

1. Actas de la Conferencia Internacional sobre Atención Primaria de Salud, Alma Ata, Kazajstán, 12 septiembre1978. Conferencia Internacional sobre Atención Primaria de Salud. Organización Mundial de la Salud. Documento A51/5.

2. Chirac P. Translating the essential drugs concept into the context of the year 2000. Trans R Soc Trop Med Hyg. 2003; 97 (1): 10-2.

3. OMS. Medicamentos esenciales. Lista Modelo de la OMS (revisada en abril de 2003) (fecha de consulta 15 septiembre de 2005). (URL disponible en http://www.who.int/medicines/organization/par/edl/expcom13/eml13_sp.pdf)

4. The selection and use of essential medicines. World Health Tech Rep Ser. 2004; 920: 1-127, back cover.

5. Lindenberg M, Kopp S, Dressman JB. Classification of orally administered drugs on the World Health Organization Midel List of Essential Medicines according to the biopharmaceutics classification system. Eur J Pharm Biopharm. 2004; 58: 265-78.

6. Hartog R. Essential and non-essential drugs marketed by the 20 largest European pharmaceutical companies in developing countries. Soc Sci Med. 1993; 37 (7): 897-904.

7. Idanpaan-Heikkila JE. Essential drugs. Ann Med. 1993; 25 (1): 19-23.

8. Guerra AA, Acurcio F de A, Gomes CA, Miralles M, Girardi SN, Werneck
GA, et al. Disponibilidade de medicamentos essenciais em duas regioes de Minas Gerais, Brasil. Rev Panam Salud Pública. 2004; 15: 168-75.

9. Molina Salazar RE, Rivas Vilchis JE. Sobreprecio y acceso a los medicamentos: el caso de los medicamentos esenciales en Mexico. Cad Saude Publica. 1998; 14 (3): 501-506.

10. Smith MK, Tickell S. The essential drugs concept is needed now more than ever. Trans R Soc Trop Med Hyg. 2003; 97 (1): 2-5.

11. Yudkin JS. Drug control: A prerequisite for health. Proc R Soc Lond Biol Sci. 1980; 209 (1174): 159-63.

12. Laupacis A, Anderson G, O'Brien B. Drug policy: Making effective drugs available without bankrupting the healthcare system. Healthc Pap. 2002; 3 (1): 12-30.

13. Laing R, Waning B, Gray A, Ford N, 't Hoen E. 25 years of the WHO essential medicines lists: Progress and challenges. Lancet. 2003; 361 (9370): 1723-9.

14. Rothberg AD, Walters L. Formulary and funding implications of the gap between the national Essential Drugs List and current prescribing in a large health maintenance organisation. S Afr Med J. 1996; 86 (9): 1084-90.

15. Howard NJ, Laing RO. Changes in the World Health Organisation Essential Drugs List. Lancet. 1991; 338 (8769): 743-5. 